\title{
Mitteilungen der Fachgesellschaften
}

Strahlenther Onkol 2013 · 189:97-97

DOI 10.1007s00066-012-0294-x

๑) Springer-Verlag Berlin Heidelberg 2013

\section{Adressen}

Deutsche Gesellschaft für Radioonkologie e.V., www.degro.org DEGRO-Geschäftsstelle, Hindenburgdamm 30, D-12200 Berlin, Telefon (+49/30) 8441-9188, Fax -9189

Präsident: Prof. Dr. J. Dunst, Universitätsklinikum Schleswig-Holstein, Klinik für Strahlentherapie, Ratzeburger Allee 160, D-23562 Lübeck,

Telefon (+49/451) 500-6661, Fax -3324

Österreichische Gesellschaft für Radioonkologie, Radiobiologie und Medizinische Radiophysik, www.oegro.net

Prim. Univ.-Prof. Dr. F. Sedlmayer, Universitätsklinik für Radiotherapie und Radio-Onkologie Salzburger Landeskliniken und Paracelsus Medizinische Privatuniversität, Müllner Hauptstraße 48, A-5020 Salzburg,

Telefon (+43/662) 4482-3904, Fax -887

Scientific Association of Swiss Radiation Oncology, www.sasro.ch PD Dr. Damien C. Weber, Service de radio-oncologie, Hôpital Cantonal Universitaire, $\mathrm{CH}$ - 1211 Genève

Hungarian Society of Radiation Oncology, www.oncol.hu Prof. Dr. A. Mayer, Center of Oncoradiology, Uzsokí Hospital, Uzsoko u. 29, H-1145 Budapest, Telefon (+36/1) 251-1471, Fax -1478,

E-Mail: mayera@hu.inter.net
Redaktion:

Prof. Dr. Rolf Sauer, Erlangen

Deutsche Gesellschaft für Medizinische Physik, www.dgmp.de Prof. Dr. P. Kneschaurek, Klinik für Strahlentherapie der Technischen Universität, Klinikum rechts der Isar, Ismaninger Straße 22, D-81675 München, Telefon (+49/89) 4140-4504, Fax -4881

Hellenic Society of Radiation Oncology, www.eeao.f2s.com Dr. P. Pantelakos, Karneadou Str. 44-46, GR-10676 Athen, Telefon/Fax (+30/1) 7244117

\section{Romanian Society of Radiotherapy and Medical Oncology,} www.srrom.ro

Prof. Dr. N. Ghilezan, 34-36, Republicii Str., 400015 Cluj-Napoca, Rumänien, Telefon (+40/264) 598361 (ext. 106),

Fax (+40/264) 598815, E-Mail: srro_server@yahoo.com

Slovak Society of Radiation Oncology, www.srobf.szm.com MUDr. Pavol Dubinský, PhD.; Východoslovenský onkologický ústav, a.s.; Rastislavova 43, 04191 Košice, Slowakei; Telefon (+421/55) 6135-501(-511,-502), Fax-504; E-Mail: dubinsky@vou.sk

\section{Erlanger Weiterbildungsveranstaltung}

\section{Simultane Radiochemotherapie}

Erlangen, 22.- 23. Februar 2013

DEGRO-Kurs im Rahmen der Weiterbildung zum Arzt für Strahlentherapie; Seminar der Deutschsprachig-Europäischen Schule für Onkologie DESO; CME-Zertifizierung durch die Bayerische

Landesärztekammer beantragt

Leitung der Veranstaltung:

Prof. Dr. R. Fietkau, Strahlenklinik, Universitätsklinikum Erlangen,

Universitätsstr. 27, 91054 Erlangen

Information und Anmeldung:

st-studiensekretariat@uk-erlangen.de,

www.strahlenklinik.uk-erlangen.de

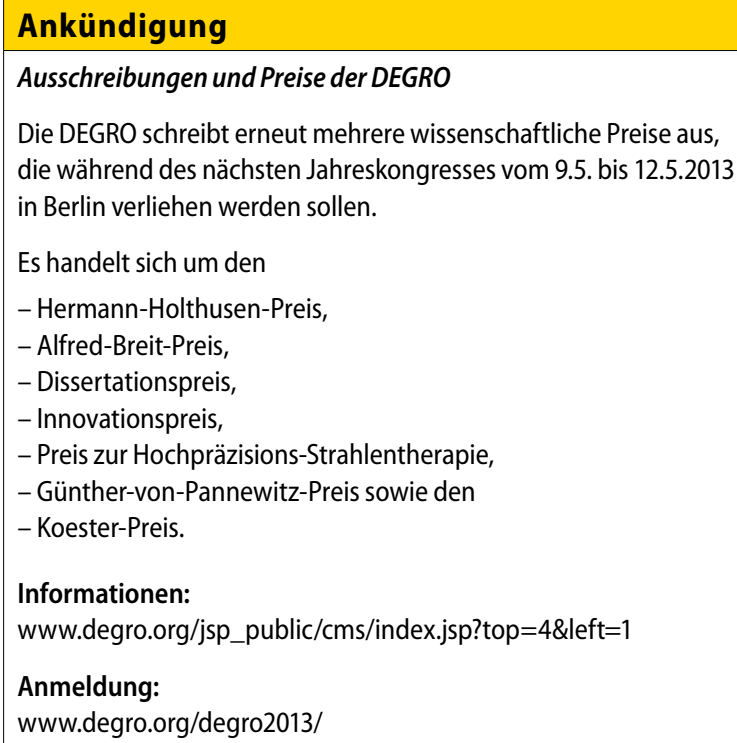

Die DEGRO schreibt erneut mehrere wissenschaftliche Preise aus, die während des nächsten Jahreskongresses vom 9.5. bis 12.5.2013 in Berlin verliehen werden sollen.

Es handelt sich um den

- Hermann-Holthusen-Preis,

- Alfred-Breit-Preis,

- Dissertationspreis,

- Innovationspreis,

- Preis zur Hochpräzisions-Strahlentherapie,

- Günther-von-Pannewitz-Preis sowie den

- Koester-Preis.

Informationen:

www.degro.org/jsp_public/cms/index.jsp?top $=4 \&$ left $=1$

Anmeldung:

www.degro.org/degro2013/ 